\title{
Liposomes as models for membrane integrity
}

Sarah J. Routledge, John A. Linney and Alan D. Goddard*

School of Life and Health Sciences, Aston University, Aston Triangle, Birmingham, B4 7ET, UK.

a.goddard@aston.ac.uk

s.routledge@aston.ac.uk

linneyj@aston.ac.uk

\begin{abstract}
Biological membranes form the boundaries to cells. They are integral to cellular function, retaining the valuable components inside and preventing access of unwanted molecules. Many different classes of molecules demonstrate disruptive properties to the plasma membrane. These include alcohols, detergents and antimicrobial agents. Understanding this disruption and the mechanisms by which it can be mitigated is vital for improved therapeutics as well as enhanced industrial processes where the compounds produced can be toxic to the membrane. This mini-review describes the most common molecules that disrupt cell membranes along with a range of in vitro liposome-based techniques that can be used to monitor and delineate these disruptive processes.
\end{abstract}

\section{Abbreviations}

$\mathrm{ABE}$, acetone-butanol-ethanol; AMPs, Antimicrobial peptides; ANTS, 8Aminonaphthalene-1,3,6-trisulfonic acid; CF, 5(6)-Carboxyfluorescein; CHAPS, 3-[(3cholamidopropyl)dimethylammonio]-1-propanesulfonate; $\mathrm{CMC}$, critical micelle concentration; CPE, conjugated polyelectrolytes; DDM, $n$-dodecyl- $\beta$-d-maltoside; DLS, dynamic light scattering; DOPC, 2-((2,3bis(oleoyloxy)propyl)dimethylammonio)ethyl hydrogen phosphate; DPX, p-xylenebis-pyridinium bromide; GP, generalised polarization; LMVs, large multilamellar vesicles; LSPR, localised surface plasmon resonance; $M \beta C D$, methyl-betacyclodextrin; NR, Nile red; PC, phosphatidylcholine; $\mathrm{PE}$, phosphatidylethanolamine; PG, phosphatidylglycerol; PI, phosphatidylinositol; PS, phosphatidylserine; RTX, repeats-in-toxin; SLBs, supported lipid bilayers; SUVs, small unilamellar vesicles; $T_{m}$, melting temperature; $\mathrm{TOCL}$, tetraoleoylcardiolipin.

\section{Introduction}

The plasma membrane is a critical component of the cell; it maintains its integrity and protects it from environmental stresses. The membrane comprises a complex bilayer of lipids, such as phospholipids, which can account for over $25 \%$ of the membrane components ${ }^{1}$, glycolipids and sterols ${ }^{2,3}$. In addition, it contains membrane proteins involved in biochemical processes, including electron and solute transport and intracellular signaling ${ }^{2}$. The integrity of the membrane is therefore essential for cell viability. 
There are various biophysical properties of the membrane which are vital for its function, including thickness, fluidity, lateral pressure, charge and permeability. Membrane fluidity is influenced by lipid composition; phospholipid bilayers are normally fluid at physiological temperatures, although their individual melting temperature $\left(T_{m}\right)$ is dependent upon the properties such as chain length, degree of unsaturation and head group shape ${ }^{4}$. Above their $T_{m}$, lipids are in a fluid liquid crystalline phase, and below this a more solid gel-phase; fluidity decreases with lower temperatures ${ }^{5,6}$. The combination of different lipids present therefore determines the fluidity of the membrane.

Membrane thickness and lateral pressure can be influenced by chain length and saturation of the lipids ${ }^{5}$. A study by Cantor $(1999)^{7}$ found that double bonds present closer to the lipid headgroups increases lateral pressure and that the presence of cholesterol influences both thickness and lateral pressure. The thickness and fluidity of membranes as well as lipid packing may also affect permeability to solutes; water permeability can be reduced by the presence of particular lipids such as cholesterol and sphinogolipids ${ }^{8}$.

The surface charge of membranes is determined by the overall charge of the cationic and anionic functional groups of the headgroups of the lipids present. Phosphatidylethanolamine (PE) and phosphatidylcholine (PC) are zwitterionic, while phosphatidylserine (PS) and phosphatidylinositol (PI) are negatively charged and are abundant in the inner leaflet; they therefore contribute significantly to the net overall negative charge of the membrane ${ }^{9}$, which in turn generates a surface potential.

Disruption of the membrane by molecular or physical methods can alter these properties and impact upon membrane stability and function. In addition, the correct function of membrane proteins is highly dependent upon their lipid environment and, therefore, alterations to the lipid components have concomitant changes on membrane protein activity. This mini-review discusses compounds that can disrupt membranes and the consequences of this upon the cell. It highlights some of the latest in vitro methods for studying membrane integrity, with a focus upon liposome-based assays that are accessible to most labs and can generally be performed without requiring specialist equipment. We have included some illustrative examples but there are many other applications of these systems across the range of membrane biology.

\section{Membrane-disrupting compounds}

\subsection{Solvents}

Organic solvents have been demonstrated to disrupt membranes; this is of significant interest to industrial process which employ microorganisms such as yeast for brewing and Clostridia species in the acetone-butanol-ethanol ( $A B E$ ) process to generate high-value chemicals ${ }^{10}$. The yields of many of these processes are hindered by the toxic effects of their products upon the membrane ${ }^{11}$. Alcohols have been 
shown to alter membrane properties such as fluidity ${ }^{12}$, permeability ${ }^{13}$ and membrane thickness ${ }^{14}$. They may affect the lateral pressure and electrostatic potential of the cell membrane, affecting cell signalling and the function of water and ion channels ${ }^{15}$. In addition, membrane disruption can lead to loss of control over solute transport and leakage of cofactors as well as reduced growth rates and viability of the cells ${ }^{2}$.

For shorter chain alcohols, the degree of partitioning into the membrane occurs according to their hydrocarbon chain length; this effect has been demonstrated to reach a plateau for longer chain alcohols ${ }^{16}$. A study by Ly and Longo $(2004)^{14}$ suggests that for every additional $\mathrm{CH}_{2}$ group, an alcohol is three times more efficient in reducing the interfacial tension of a bilayer and that three times more alcohol partitions into the bilayer-water interface. Molecular dynamics simulations by Patra et al (2006) $)^{15}$ demonstrated that ethanol readily penetrates the membrane and appears to localize close to ester oxygens of lipids and hydrogen bonds with them. This was not the case with the more polar molecule methanol, which was unable to cross the membrane, nor form hydrogen bonds. Ethanol was found to increase the area per lipid and to decrease bilayer thickness, and as a result, the membrane becomes more permeable to small molecules and impacts upon the function of membrane proteins.

Organisms such as E. coli are thought to compensate for the effects of alcohol by altering their membrane lipid composition to increase rigidity in the presence of ethanol, where they replaced $\mathrm{C} 16: 0$ chains with $\mathrm{C} 18: 1^{17}$. Clostridia species have been demonstrated to compensate for increased fluidity by increasing the quantity of saturated lipids in the membrane ${ }^{18}$. This enables the lipids to pack together more tightly than unsaturated lipids, decreasing fluidity. In vitro studies on the effect of specific lipids on membrane resistance to alcohols could help to improve the efficiency of numerous biotechnological processes.

\subsection{Detergents}

Detergents are amphipathic surface-active molecules. In aqueous environments they spontaneously form micelles due to their polar head groups and hydrophobic tails ${ }^{19}$. They are grouped into ionic detergents e.g. sodium dodecyl sulfate (SDS), anionic detergents e.g. n-Dodecyl $\beta$-D-maltoside (DDM) and zwitterionic detergents e.g. 3[(3-cholamidopropyl)dimethylammonio]-1-propanesulfonate (CHAPS), and their properties are dependent upon their structure ${ }^{20}$. They are able to disrupt cell membranes at concentrations above their critical micelle concentration (CMC), and this effect is frequently utilised to release membrane proteins from their lipid environment for further biophysical studies due to formation of protein-detergent complexes $^{20}$.

Solubilisation of lipid membranes is complex, however it is thought to occur in three stages. The first stage occurs at low detergent concentrations, and the detergent partitions between the aqueous medium and the membrane with little disruption. The term $\mathrm{R}_{e}^{\text {sat }}$ is used describe the lipid to detergent ratio necessary for the onset of 
solubilisation, and above this the second stage of solubilisation occurs; increased concentrations of detergent disrupt the bilayer to form mixed micelles containing lipids, protein and detergent. At higher concentrations of detergent the third stage occurs, where the protein-lipid-detergent micelles mix rapidly with detergent micelles and the bilayer is entirely solubilised $\left(R_{e}^{\text {sol }}\right)$. Smaller micelles of lipid and detergent are produced, with a higher detergent to lipid ratio. This involves stripping away lipid from the protein to produce lipid-detergent and protein-detergent mixed micelles. $D_{w}{ }^{\text {sat }}$ and $D_{w}{ }^{\text {sol }}$ are the values for the detergent concentration at the initiation of saturation and solubilisation, and can be back-extrapolated by measuring turbidity at different concentrations of lipid ${ }^{21,22}$.

A range of surfactants and polymers such as fluorinated surfactants ${ }^{23}$, styrene-maleic acid copolymers ${ }^{24}$ and amphiphipols ${ }^{25}$ are being explored for improvements over traditional detergents. The development of surfactants able to extract membrane proteins from their lipid environment more efficiently and better maintain their stability will greatly aid the study of these proteins.

\subsection{Local anaesthetics}

Local anaesthetics used to achieve anaesthesia and analgesia are known to influence membrane integrity. They consist of a lipophilic aromatic ring, an intermediate ester or amide chain and a terminal amine. Their lipid solubility allows them to diffuse into neural membranes to bind and disrupt the action of sodium channels, preventing depolarisation ${ }^{26}$. In general, the more hydrophobic the molecule, the more efficient the effect. However, this also leads to greater toxicity. This is thought to be due to non-specific action of these drugs upon the membrane, where at high concentrations they increase membrane permeability and fluidity ${ }^{27}$. Some local anaesthetics such as bupivacaine have been reported to have a negative effect upon the heart ${ }^{27}$; a better understanding of how these drugs alter membrane fluidity could help to reduce side effects.

\subsection{Antimicrobial agents}

Cell membranes are the target of numerous antimicrobial agents, which are often lipophilic. They can interact directly with the membrane to disrupt its integrity, and may as a consequence disrupt the function of membrane proteins ${ }^{28}$. Drugs able to disrupt the bacterial membrane include the lipoglycopeptides daptomycin, telavancin, oritavancin and dalbavancin. They function by interacting with negatively charged and zwitterionic phospholipids. For example, oritavancin has a net positive charge, and it disrupts membranes abundant in phosphatidylglycerol (PG) and cardiolipin ${ }^{28}$. Studies on antimicrobial agents such as shikimic acid have suggested that it is able to cause leakage of $\mathrm{K}^{+}$and nucleotides from Staphylococcis aureus by interacting with both membrane lipids and proteins, decreasing membrane fluidity and increasing its permeability, ultimately inhibiting growth and leading to cell death $^{29}$. 
Antimicrobial peptides (AMPs) are molecules usually ranging from 12-50 amino acids and their secondary structures include $\alpha$-helix, $\beta$-sheet and loops ${ }^{30,31,32}$. Many AMPs are amphipathic ${ }^{30,31}$ which has been shown to be an important factor in their ability to bind to membranes ${ }^{32,33}$. Although the precise mechanism of action of many AMPs is still unknown, several studies have suggested that their properties allow them to cross the cell wall or outer membrane and disrupt it, leading to cell death ${ }^{30}$. In contrast, some AMPs penetrate the membrane without significantly disrupting it to interact with intra cellular targets leading to cell death ${ }^{34,35}$. Many AMPs are cationic and are rich in positively charged amino acids such as lysine and arginine, which allow the peptides to interact with negatively charged lipids in bacterial membranes, driving the first step in insertion and antibacterial activity. They may also contain hydrophobic residues such as tryptophans or phenylalanines. These amino acids contribute to the ability of the peptides to interact with and disrupt bacterial membranes ${ }^{30}$. Simulations have been performed for AMPs such as protegin-1 interacting with patches of bilayer ${ }^{36}$. The simulations showed that depending upon the peptide to lipid ratio, PG-1 forms different structures leading to membrane disruption. In the model, PG-1 was able to bind to the edge of the bilayer, form pores by causing local curvature, and at higher peptide to lipid ratios formed wormlike micelles ${ }^{36}$. Other AMPs are thought to disrupt membranes with a detergent-like "carpet" mechanism such as cecropin A (Fig. 1). The peptides are thought to build up at the membrane surface until they reach a critical concentration where it is covered like a carpet; they then insert into the membrane, disrupting it without the formation of pores ${ }^{31}$. Another group of cytotoxic molecules are poreforming toxins, which are produced by bacteria and some eukaryotes. These proteins that associate with target cell membranes to form pores that destroy the integrity of the cell ${ }^{37}$.

In summary, numerous molecules are able to disrupt membranes with several mechanisms of action; alcohols can insert into membranes to increase the area per lipid $^{15}$, and like anaesthetics, alter fluidity ${ }^{27}$, impacting on the function of membrane proteins. Some antimicrobial agents are thought to act in a similar manner to detergents and disrupt the membrane to form micelle-like structures ${ }^{31}$. Other AMPs can form pores, as can pore-forming toxins, potentially leading to osmotic swelling and Iysis ${ }^{37}$. Continued in vitro studies are still needed to further enhance our understanding of the mechanism of action of many of these important agents.

\section{Methods to investigate the membrane integrity of liposomes}

Many in vitro studies on membranes utilise liposomes. Liposomes are spherical phospholipid bilayers enclosing an internal aqueous environment. Because of this similarity with cellular membranes, they are often used as simple cell models ${ }^{38}$. Additionally, because of their ability to compartmentalise their internal aqueous environment from the external environment, they can be used in drug delivery and membrane integrity assays ${ }^{39}$.

The lipid composition of liposomes depends on the desired model, for example a molar ratio of 3 phosphatidylethanolamine: 1 phosphatidylglycerol is considered a 
good model of an inner bacterial membrane ${ }^{40}$. In contrast, eukaryotic membranes are predominantly phosphatidylcholine, phosphatidylethanolamine, phosphatidylserine and phosphatidylinositol with phosphatidylglycerol being a minor species. There is also variation between subcellular membrane compositions. For example, in Saccharomyces cerevisiae the plasma membrane is primarily phosphatidylserine whereas the mitochondrial membrane is mainly phosphatidylcholine ${ }^{41}$. Conventional liposome models will have identical leaflets comprising the bilayer, which is not reflective of the diversity seen in biological membrane. Recently, a novel protocol has been developed to produce liposomes with asymmetric leaflet compositions utilising methyl- $\beta$-cyclodextrin-catalyzed exchange between liposome populations of different lipid compositions ${ }^{42}$. This method is more versatile than previous methods such as enzymatic headgroup modification ${ }^{43}$ or redistribution of charged lipids by changing the $\mathrm{pH}^{44}$, both of which can only work with a specific population of lipids.

The procedure for making liposomes is relatively simple ${ }^{45}$ and relies on hydrophobic interactions between lipid molecules and an aqueous solution (Fig. 2). A mixture of lipid in organic solvent is added to a round-bottom flask. The solvent is evaporated with an inert gas, such as $\mathrm{N}_{2}\left(\mathrm{O}_{2}\right.$ exposure is to be avoided to prevent lipid oxidation) to produce a thin lipid film around the bottom of the flask. Following this, the lipid film is rehydrated with an aqueous solution (which can contain dye / drug molecules) upon which the lipid film will spontaneously self-assemble into liposomes ${ }^{46}$. The choice of resuspension buffer will depend on the application but is generally tris or phosphate-buffered saline at a temperature above that of the $T_{m}$ of the lipid mixture. The presence of counter ions in the buffer can aid liposome formation and prevents aggregation. The $\mathrm{pH}$ will depend on the desired ionisation of headgroups present.

Rehydration forms a heterogeneous mixture of multilamellar liposomes, which can range in size - from this, several different liposome classes can be made. Giant unilamellar vesicles (GUV) (1-100 $\mu \mathrm{m})$ are used as eukaryotic cell models. Early protocols for GUV formation involved adding aqueous buffer to a thin layer of hydrated phospholipids and allowing them to swell ${ }^{47}$. However, this method is time consuming and often produces a high proportion of large multilamellar vesicles $(\mathrm{LMV})^{48}$. To speed up this process, lipids can be deposited on electrodes. The swelling process is enhanced following the application of an electric field ${ }^{49}$. A population of smaller unilamellar liposomes can be produced through use of sonic force (sonication) or mechanical force (extrusion). Extrusion is the process of passing the liposome suspension through a polycarbonate filter of a define pore size $>10$ time. By using filters of different sizes, large unilamellar vesicles (LUV) (200-1000 $\mathrm{nm}$ ) and small unilamellar vesicles (SUV) ( $\geq 100 \mathrm{~nm}$ ) can be produced. LUVs can be used as bacterial cell models and SUVs can mimic extracellular vesicles ${ }^{50}$. The size of the liposome produced affects the curvature of the bilayer, where smaller SUVs have higher membrane curvature than LUVs ${ }^{51}$. Curvature can influence the ability of some proteins to bind ${ }^{52}$ and affects lipid packing ${ }^{51}$. The bilayer of GUVs are thought to posses more similar physical properties to the bilayer of cells ${ }^{53}$; it is therefore necessary to consider the size and curvature of the liposome most appropriate for 
the investigation. Freeze-thawing of multilamellar vesicles is often employed to reduce the lamellarity of the vesicles whilst also improving their encapsulation efficiency ${ }^{54,55}$. Methods of liposome formation have been reviewed previously ${ }^{56,57}$.

\subsection{DLS}

Dynamic light scattering (DLS) is a technique used to calculate the size distribution of particles in a solution. It can be applied to in vitro membrane stability assays to determine the diameter of membrane models and how they change over time in response to varying conditions. DLS utilises the Brownian motion of the sample particles to calculate their diameter ${ }^{58}$. Initially a monochromatic beam of light is fired at the sample, the light then subsequently undergoes Rayleigh scattering provided the particles are smaller than $1 / 10^{\text {th }}$ of the incident light wavelength ${ }^{59}$ (as shown in Fig. 3). The intensity of this scattering varies over time due to the movement of particles. The fluctuations in intensity of the scattered light are measured and used to determine the Brownian motion. This can then be used in conjunction with the Stokes-Einstein relationship to yield particle diameter ${ }^{59}$. DLS readings can be conducted rapidly with the majority of the process being automated. Additionally, DLS can be performed on a range of different sized particles and low concentrations. A drawback of DLS is that it assumes that particles are of a spherical shape, limiting its accuracy when measuring non-spherical samples ${ }^{60}$.

\subsubsection{Examples of use}

DLS has been employed in several studies investigating membrane integrity and stability in response to various agents. For example, Liu and Liu $(2017)^{61}$ have used DLS to show how the diameter of choline phosphate (inversed phosphocholine) liposomes increased upon exposure to $\mathrm{Zn}^{2+}$, suggesting swelling or fusion. DLS has been used to monitor membrane integrity in response to conjugated polyelectrolytes (CPE). CPEs are polymers containing delocalised electron systems which can be used as fluorescent membrane markers. They have advantageous properties compared to conventional fluorescent dyes and quantum dots - being nontoxic and photostable whilst retaining sensitivity. Their interactions with the cell membrane - whether they caused liposome fusion and decomposition - were investigated using liposome models and $\mathrm{DLS}^{62}$.

\subsection{Nanoplasmonic sensing}

Nanoplasmonic sensing is a powerful optical technology, which can provide real-time insights into structural changes of biomembranes, interfacial interactions as well as lipid bilayer and liposome stability ${ }^{63}$. The technique can provide highly sensitive information regarding the shape, conformation and thickness of molecules of interest without the need for labels. Additionally, how these variables are affected 
by changing external parameters such as solution $\mathrm{pH}$ or presence of a membranedisrupting compounds and can also be investigated ${ }^{64}$.

Samples are adsorbed onto a metal-coated array, usually gold due to its inert nature (Fig. 4$)^{64}$. As a result of incident visible-light photons interacting with nanoscale metallic structures, the free electrons in the metal begin to oscillate locally. This results in localised surface plasmon resonance (LSPR) and the maximum light extinction wavelength $\left(\lambda_{\max }\right)^{65}$. Structural changes to adsorbed molecules (e.g. liposomes or bilayers) within close proximity to these oscillating electrons can cause changes in the refractive index of the local environment ${ }^{66}$. This subsequently leads to $\lambda_{\max }$ to shift, which is the primary measurement in nanoplasmonic sensing ${ }^{67,68}$ Liposome deformation can be detected by a red shift in the $\lambda_{\max }$. This occurs due to a change in the refractive index because the adsorbed liposome begins to spread along the nanoparticle surface when it deforms ${ }^{69}$. As the array is based on a surface, the use of LSPR can be combined with spectroscopic techniques such as infrared, Raman and various forms of microscopy ${ }^{70}$.

\subsubsection{Examples of use}

Nanoplasmonic sensing has been used to investigate membrane curvature ${ }^{71}$ and how this affects membrane-protein interactions as well as the role of cholesterol in membrane stability ${ }^{1}$. The disruptive effects of amphiphillic compounds such as detergents upon small unilamellar vesicles ${ }^{72}$, and the effects of ionic liquids upon liposomes have also been investigated using this technique ${ }^{1}$. Recent research has also analysed lipid bilayers supported on silica-coated nanowell and nanodisk sensing platforms ${ }^{72}$. Jonsson and others (2007) were able to show a characteristic change in the LSPR signal following vesicle rupture leading to the formation of a SLB using a gold nanoplasmonic film on $\mathrm{a} \mathrm{SiO}_{2}$ encapsulated nanohole ${ }^{73}$. The effect of osmotic pressure on the deformation of fluid-phase and gel-phase liposomes was investigated using nanoplasmonics. It was found that increasing osmotic pressures resulted in deformation for fluid-phase vesicles only ${ }^{69}$. Additionally, Oh and others $(2015)^{66}$, found that increasing temperature to alter lipid phase states had varying effects on the structure of adsorbed liposomes.

\subsection{Dye leakage assays}

Dye leakage assays are a staple technique for reviewing membrane stability and integrity in vitro. They can provide a quantitative measurement of membrane disruption and hence have important applications when investigating the cytotoxicity of a novel compound. Some of these dyes and their uses are summarised in Table 1. Generally, the use of fluorescent dyes is employed to assay membrane integrity. The dye will be unable to transverse the membrane under normal conditions and will be present on only one side of a bilayer (e.g. inside a liposome) at a concentration sufficient to cause self-quenching of the fluorescence. Following disruption to the membrane by permeabilisation or rupture, the dye will leak out where it can be detected by fluorescent spectroscopy. An increase in fluorescence will indicate instability in the membrane under investigation. A 
drawback of using dye leakage assays alone is the inability to differentiate between increased permeabilisation and total liposome lysis. Therefore, these assays can be combined with DLS to enable detection of lysis in addition to the changes in permeabilisation.

An example of this is the use of 5(6)-Carboxyfluorescein (CF), a fluorescent dye which self-quenches fluorescence at high concentrations (>97\% of fluorescence is quenched at $0.2 \mathrm{M})^{74}$. This self-quenching can be exploited in membrane integrity assays as illustrated in Fig. 5. A solution of highly concentration CF can be used to rehydrate a lipid film resulting in spontaneous liposome formation encapsulating the CF dye molecules inside. Following a series of washes to remove the excess CF molecules in the surrounding buffer, a solution of CF-containing liposome in clean buffer is yielded. These liposomes can then be exposed to antimicrobial, solvents or other compounds to assay their impact on the integrity of membranes.

Table 1: Dyes commonly utilised in vesicle dye-release assays

\begin{tabular}{|l|l|l|l|l|}
\hline \multicolumn{1}{|c|}{ Dye } & \multicolumn{1}{|c|}{ Application } & Wavelengths & Measurement & Reference \\
\hline ANTS and DPX & $\begin{array}{l}\text { Effect of RTX proteins } \\
\text { upon liposome integrity. }\end{array}$ & $\begin{array}{l}\text { Excitation } 370 \mathrm{~nm} \\
\text { Emission } 505 \mathrm{~nm}\end{array}$ & Fluorescence & $\begin{array}{l}\text { Fišer and } \\
\text { Konopásek } \\
(2009)^{75}\end{array}$ \\
\hline Calcein & $\begin{array}{l}\text { Effect of local } \\
\text { anaesthetics upon } \\
\text { liposome integrity. }\end{array}$ & $\begin{array}{l}\text { Excitation } 490 \mathrm{~nm} \\
\text { Emission } 520 \mathrm{~nm}\end{array}$ & Fluorescence & $\begin{array}{l}\text { Efimova } \text { et } \\
\text { al (2016) }\end{array}$ \\
\hline Carboxyfluorescein & $\begin{array}{l}\text { Effect of AMPs upon } \\
\text { liposome integrity. }\end{array}$ & $\begin{array}{l}\text { Excitation } 480 \mathrm{~nm} \\
\text { Emission } 510 \mathrm{~nm}\end{array}$ & Fluorescence & $\begin{array}{l}\text { Ambroggio } \\
\text { et al } \\
(2005)^{76}\end{array}$ \\
\hline Doxorubicin & $\begin{array}{l}\text { Characterisation of } \\
\text { temperature sensitive } \\
\text { liposomes for } \\
\text { intravascular triggered } \\
\text { drug release. }\end{array}$ & $\begin{array}{l}\text { Excitation } 650 \mathrm{~nm} \\
\text { Emission } 673 \mathrm{~nm}\end{array}$ & Fluorescence & $\begin{array}{l}\text { Burke } \text { et al } \\
(2018)^{77}\end{array}$ \\
\hline FITC-dextran & $\begin{array}{l}\text { Investigation into trans- } \\
\text { activating } \\
\text { transcriptional activator } \\
\text { protein mediated } \\
\text { transduction of } \\
\text { liposome contents into } \\
\text { cells. }\end{array}$ & $\begin{array}{l}\text { Excitation } 494 \mathrm{~nm} \\
\text { Emission } 518 \mathrm{~nm}\end{array}$ & Fluorescence & $\begin{array}{l}\text { Torchillin } \text { et } \\
\text { al (2001) }\end{array}$ \\
\hline Methylene blue & $\begin{array}{l}\text { Characterisation of } \\
\text { liposomes in } \\
\text { polymersomes. }\end{array}$ & Excitation $633 \mathrm{~nm}$ & UV-visible & $\begin{array}{l}\text { Peyret } \text { et al } \\
(2017)^{79}\end{array}$ \\
\hline
\end{tabular}




\subsubsection{Examples of use:}

Dye release assays can provide information about the mechanism of toxicity of novel compounds. Membrane lysis in response to several antimicrobial peptides from Australian tree frogs has been investigated using CF-encapsulated liposomes ${ }^{76}$. Model membranes composed of 1-palmitoyl-2-oleoyl-sn-glycero-3-phosphocholine (POPC) and 1-palmitoyl-2-oleoyl-sn-glycero-3-phospho-(1'-rac-glycerol) (POPG) lipids were treated with peptides Maculatin, Citropin and Aurein. It was found that increasing the molar ratio of antimicrobial:lipid resulted in more dye leakage suggesting a greater degree of membrane permeabilisation.

The effects of two synthetic antimicrobials, namely bicarinalin and BP100 were investigated by Eales and colleagues $(2018)^{80}$. CF-containing liposomes were made from the extracted lipids of Acinetobacter baumannii, an opportunistic pathogen which is often resistant to a range of antimicrobial treatments. Based on CF leakage from these liposomes, the authors concluded the mechanism of action of bicarinalin and BP100 to be via membrane pore formation. This was because a higher level of relative fluorescence was seen with increasing concentrations of antimicrobial agent, suggesting they cause dye leakage from liposomes.

8-Aminonaphthalene-1,3,6-trisulfonic acid (ANTS) is an anionic fluorophore and p-xylene-bis-pyridinium bromide (DPX) is a cationic quencher. They can be combined in a pair to investigate membrane stability ${ }^{81}$. Fišer and Konopásek (2009) ${ }^{75}$ used this combination to determine and compare the pore-forming effects of bacterial repeats-in-toxin (RTX) proteins on liposome integrity. Specifically, whether these toxins resulted in narrow pore formation and a "graded leakage" or larger pores and an "all-or-nothing leakage."

\section{4 Polarity sensitive probes}

Probes sensitive to polarity are commonly used to measure changes membrane fluidity and lipid packing by determining the generalised polarization (GP). The emission spectra of these probes shifts depending upon the polarity of the bilayer environment ${ }^{82,83}$. This can be influenced by the degree of hydration of the membrane, where fewer water molecules may be present in closely packed membranes consisting of saturated lipids, whereas greater numbers of water molecules are usually present in unsaturated membranes ${ }^{67}$.

Commonly used probes include laurdan, which has an emission maximum of $440 \mathrm{~nm}$ in more rigid, tightly packed membranes and $490 \mathrm{~nm}$ in more fluid membranes. It is therefore an ideal probe to measure changes in membrane phase transitions and integrity ${ }^{82,83}$. Other probes include di-4-ANEPPDHQ with emission maxima of $560 \mathrm{~nm}$ in gel phase membranes and $610 \mathrm{~nm}$ in liquid phase membranes ${ }^{83}$. Nile red (NR), 9- 
diethylamino-5H-benzo[alpha]phenoxazine-5-one, has also been used to measure membrane properties ${ }^{84}$.

\subsubsection{Examples of use:}

Laurdan has been used to study dipalmitoylphosphatidylcholine (DPPC) multilamellar vesicles containing different concentrations of cholesterol to investigate phospholipid order and fluidity ${ }^{66}$. The study utilised laurdan GP and anisotropy to assess changes in fluidity with increasing temperature, and demonstrated a decrease in both GP and anisotropy with increasing temperature. The probe was able to report on the transition of DPPC from gel to liquid crystalline states. Similar studies have also been performed upon sphingomyelin liposomes with cholesterol content ranging from $0 \%$ to $50 \mathrm{~mol} \%{ }^{85}$. Laurdan detected an increase in fluidity of the membranes with temperature, shown by a decrease in GP, and the addition of cholesterol reduced the magnitude of the drop in GP. The study found that the probe was able to report the phase transition from liquid-ordered to liquiddisordered, however did not detect transition from the solid-ordered to liquidordered.

\subsection{Imaging}

The visual morphology of liposome models can provide important insights into their integrity. Fluorescent microscopy can elucidate various aspects of liposomes including shape and size, however the addition of the fluorescent probe may inadvertently alter liposome characteristics hence the selection of an appropriate probe is paramount ${ }^{86}$.

Unlike fluorescent microscopy, confocal microscopy can eliminate out of focus signal by utilising a focused beam of light on a small part of the sample, leading to a higher resolution. Additionally, 3D images can also be produced by scanning the sample, thus providing a great deal of structural information ${ }^{87}$. In the past decade, confocal imaging has been combined with a new class of probes termed, fluorescent flippers which can be used to monitor the lateral pressure of $\mathrm{GUVs}^{88,89}$. The fluorescent lifetime of these probes depends on the twist between the fluorescent groups, which correlates to lateral pressure ${ }^{90}$. Whilst confocal microscopy can provide high quality images of GUVs, high definition images of SUVs are often unobtainable ${ }^{91}$.

Cryogenic electron microscopy (Cryo-EM) is type of microscopy in which samples are applied to a grid and then rapidly frozen (usually with ethane) before imaging. It is possible to visualise a liposome's shape, size and any internal structures ${ }^{92}$. Furthermore, cryo-EM avoids structural alterations stemming from sample preparation that are seen in transmission electron microscopy ${ }^{93}$. Cryo-EM has been used to elucidate the mechanism of propranolol on liposomes - showing disruption to lipid organisation in the bilayer ${ }^{94}$.

\subsection{Other}


Differential scanning calorimetry (DSC) measures the specific heat capacity of physical transformations (e.g. phase transitions) which are induced by heat ${ }^{95}$, therefore DSC can be used to investigate liposomes. DSC provides thermodynamic information of liposomes, such as their $T_{m}$ associated enthalpies, the interactions of compounds with liposomes and liposome fusion ${ }^{96-98}$. Small-angle X-ray scattering (SAXS) is another technique which can be used to investigate liposome fusion ${ }^{99}$. In SAXS a monochromatic X-ray beam is fired at a sample and subsequently undergoes scattering once it hits the sample. The intensity of scattered electrons at a particular angle are measured. Scattered X-rays contain structural information about the size, shape and surface area to volume ratio ${ }^{99}$. The location of compounds within the bilayer can be determined, for example cholesterol ${ }^{100}$ and potentially other compounds which insert into and disrupt the bilayer.

\section{Conclusions}

A range of methods are now available for studying liposomes as simple membrane models; dye leakage assays can provide information on their disruption and probes can indicate changes to fluidity. Real-time data can be gathered using nanoplasmonic sensing to determine the conformation, curvature and thickness of liposomes, and changes in size can be measured by DLS. Combined, these liposomebased techniques are simple but powerful tools to investigate membrane integrity. Many of these techniques are key in examining the effects of potentially toxic compounds such as solvents and lipophilic drugs upon the cell membrane, and allow us to better understand how cells adapt to these challenges and how the membrane is affected in disease states.

\section{Perspectives}

- Liposome-based techniques provide crucial information on how drugs, antimicrobial agents and solvents interact with the cell membrane. They allow defined lipid compositions and sizes to be investigated and are a versatile and important tool for the study of membrane integrity.

- Current easily-accessible techniques to study liposomes include DLS, nanoplasmonic sensing and the use of dyes and polarity sensitive probes. These studies have illustrated how temperature, osmotic pressure and cholesterol content affect membrane properties and have shed light upon the mechanisms of action of antimicrobial agents and anaesthetics via pore formation.

- Liposomes have many applications as simple cell membrane models, and the range of methods to characterize them is growing; new probes to monitor their properties are regularly being developed. The study of liposomes will continue to have a major impact upon our understanding of cell membranes and how therapeutic molecules and industrial chemicals disrupt them.

\section{Acknowledgements}

The authors are grateful for the support of the BBSRC (BB/R02152X/1). 


\section{Declarations of interest}

The authors declare that they have no competing interests.

\section{Authors' contributions}

All authors contributed to, read and approved the final version of the manuscript.

\section{References}

1. Russo, G., Witos, J., Rantamäki, A.H., and Wiedmer, S.K. (2017) Cholesterol affects the interaction between an ionic liquid and phospholipid vesicles. A study by differential scanning calorimetry and nanoplasmonic sensing Biochim Biophys Acta, 1859(12): 2361-2372.

2. Huffer, S., Clark, M. E., Ning, J. C., Blanch, H, W., and Clark, D,S. (2011) Role of alcohols in growth, lipid composition, and membrane fluidity of yeasts, bacteria, and archaea. Appl Environ Microbiol, 77(18): 6400-8.

3. Van Meer G., Voelker D. R., and Feigenson G., W. (2008) Membrane lipids: where they are and how they behave. Nat Rev Mol Cell Biol. 9(2): 112-124.

4. Dowhan, W and Bogdanov, M. Functional roles of lipids in membranes (2002) Biochemistry of Lipids, Lipoproteins and Membranes $\left(4^{\text {th }}\right.$ Ed) Elservier Science.

5. Eze, M. O. Phase Transitions in Phospholipid Bilayers: Lateral Phase Separations Play Vital Roles in Biomembranes (1991) Biochemical Education, 19(4).

6. Losa, A. D., and Muratab, N. (2004) Membrane fluidity and its roles in the perception of environmental signals Biochimica et Biophysica Acta, 1666: 142-157.

7. Cantor, R, S. (1999) Lipid Composition and the Lateral Pressure Profile in Bilayers Biophys J, 76(5): 2625-2639.

8. Mathai, J. C., Tristram-Nagle, S., 2 Nagle, J. F., and Zeidel, M., L. (2008) Structural Determinants of Water Permeability through the Lipid Membrane J Gen Physiol, 131(1): 69-76.

9. Goldenberg, N., M. and Steinberg, B., E. (2010) Surface Charge: A Key Determinant of Protein Localization and Function Cancer Res, 70(4).

10. Ezeji T.C., Qureshi, N. and Blaschek, H.P. (2007) Bioproduction of butanol from biomass: from genes to bioreactors Current Opinion in Biotechnology, 18(3): 220227.

11. Buehler, E. A., and Mesbah, A. (2016) Kinetic Study of Acetone-Butanol-Ethanol Fermentation in Continuous Culture PLoS One, 11(8): e0158243. 
Chen, R.F. and Knutson, J.R. (1988) Mechanism of fluorescence concentration quenching of carboxyfluorescein in liposomes: Energy transfer to nonfluorescent dimers Analytical Biochemistry, 172(1): 61-77.

12. Chin, J. H., and D. B. Goldstein (1977) Effects of low concentrations of ethanol on the fluidity of spin-labelled erythrocyte and brain membranes. Mol. Pharmacol, 13: 435-441.

13. Komatsu, H. and Okada, S. (1997) Effects of Ethanol on Permeability of Phosphatidylcholine/Cholesterol Mixed Liposomal Membranes Chemistry and Physics of Lipids, 85; 67-74.

14. Ly, H. V., and Longo, M. L. (2004) The Influence of Short-Chain Alcohols on Interfacial Tension, Mechanical Properties, Area/Molecule, and Permeability of Fluid Lipid Bilayers Biophys J 87(2): 1013-1033.

15. Patra, M., Salonen, E., Terama, E., Vattulainen, I., Faller, R., Lee, B. W., Holopainen, J., and Karttunen, M. (2006) Under the Influence of Alcohol: The Effect of Ethanol and Methanol on Lipid Bilayers Biophys J, 90(4): 1121-1135.

16. Ingólfsson, H, I., and Andersen, O. S. (2011) Alcohol's Effects on Lipid Bilayer Properties Biophys J, 101(4): 847-855.

17. Dombek, K, M., and Ingram L. O. (1984) Effects of Ethanol on the Escherichia coli Plasma Membrane J Bacteriol, 157(1): 233-239.

18. Baer, S.H., Blaschek, H. P., and Smith T. L. (1987) Effect of Butanol Challenge and Temperature on Lipid Composition and Membrane Fluidity of Butanol-Tolerant Clostridium acetobutylicum Appl Environ Microbiol, 53(12):2854-61.

19. Seddon, A. M., Curnow, P., Booth, P. J. (2004) Membrane proteins, lipids and detergents: not just a soap opera Biochim Biophys Acta, 3;1666(1-2): 105-17.

20. Garavito, R. M., Ferguson-Miller, S. (2001) Detergents as Tools in Membrane Biochemistry J Biol Chem, 31;276(35): 32403-6.

21. Lichtenberg, D., Ahyayauch, H., and Goñi, F. M. (2013) The Mechanism of Detergent Solubilization of Lipid Bilayers Biophys J 105(2): 289-299.

22. Kalipatnapu, S. and Chattopadhyay, A. (2005) Membrane Protein Solubilization: Recent Advances and Challenges in Solubilization of Serotonin ${ }_{1 A}$ Receptors IUBMB Life, 57(7): $505-512$.

23. Boussambe, G. N. M., Guillet, P., Mahler, F., Marconnet, A., Vargas, C., Cornut, D., Soulié, M., Ebel, C., Le Roy, A., Jawhari, A., Bonneté, F., Keller, S., and Durand, G. (2018) Fluorinated diglucose detergents for membrane-protein extraction Methods, 147: 84-94. 
24. Giusti, F., Popot, J-L., and Tribet, C. (2012) Well-defined critical association concentration and rapid adsorption at the air/water interface of a short amphiphilic polymer, amphipol A8-35: a study by Förster resonance energy transfer and dynamic surface tension measurements. Langmuir, 28:10372-10380.

25. Jamshad, M., Charlton, J., Lin, Y-P., Routledge, S. J., Bawa, Z., Knowles, T. J., Overduin, M., Dekker, N., Dafforn, T. R., Bill, R. M., Poyner, D. R., and Wheatley, M. (2015) G-protein coupled receptor solubilization and purification for biophysical analysis and functional studies, in the total absence of detergent Biosci Rep, 35(2).

26. Becker, D. E., and Reed, K. L. (2006) Essentials of Local Anesthetic Pharmacology Anesth Prog, 53(3): 98-109.

27. Efimova, S. S., Medvedev, R.Y., Schagina, L.V., Ostroumova, S. (2016) An increase in model lipid membrane fluidity as a result of local anesthetic action Cell Tiss. Biol, 10: 324.

28. Hurdle, J.G., O'Neill, A.J., Chopra, I., and Lee, R.E. (2011) Targeting bacterial membrane function: an underexploited mechanism for treating persistent infections Nat Rev Microbiol, 9(1): 62-75.

29. Bai, J., Wu, Y., Liu, X., Zhong, K., Huang, Y., and Gao, H. (2015) Antibacterial Activity of Shikimic Acid from Pine Needles of Cedrus deodara against Staphylococcus aureus through Damage to Cell Membrane Int J Mol Sci, 16(11): 27145-27155.

30. Lee, J. and Lee, D. G. (2015) Antimicrobial Peptides (AMPs) with Dual Mechanisms: Membrane Disruption and Apoptosis J. Microbiol. Biotechnol, 25(6): 759-764.

31. Sato, H., and Feix, J. B. (2006) Peptide-membrane interactions and mechanisms of membrane destruction by amphipathic $\alpha$-helical antimicrobial peptides Biochim Biophys Acta, 1758: 1245-1256.

32. Bahar, A. A., and Ren, D. (2013) Antimicrobial Peptides Pharmaceuticals, 6(12): 1543-1575.

33. Fernandez-Vidal, M., Jayasinghe, S., Ladokhin, A. S., and White, S. H. (2007) Folding amphipathic helices into membranes: Amphiphilicity trumps hydrophobicity J. Mol. Biol, 370:459-470.

34. Henriques, S. T., Melo, M. N., and Castanho, M. A. R. B. (2006) Cell-penetrating peptides and antimicrobial peptides: how different are they? Biochem J, 1; 399: 1-7.

35. Park, C. B., Kim, H. S., and Kim, S. C. (1998) Mechanism of action of the antimicrobial peptide buforin II: buforin II kills microorganisms by penetrating the cell membrane and inhibiting cellular functions Biochem Biophys Res Commun, 
244(1):253-7.

36. Lam, K. L. H., Ishitsuka, Y., Cheng, Y., Chien, K., Waring, A. J., Lehrer, R. I., and Lee, K. Y. C. (2006) Mechanism of Supported Membrane Disruption by Antimicrobial Peptide Protegrin-1 J. Phys. Chem. B, 110: 21282-21286.

37. Cabezas, S., Ho, S., Ros, U., Lanio, M. E., Alvarez, C., Van der Goot, F. G. (2017) Damage of eukaryotic cells by the pore-forming toxin sticholysin II: Consequences of the potassium efflux. Biochim Biophys Acta Biomembr,1859(5):982-992.

38. Matos, C., Moutinho, C., and Lobão, P. (2012) Liposomes as a model for the biological membrane: Studies on daunorubicin bilayer interaction Journal of Membrane Biology, 245(2): 69-75.

39. Hernández, V., Karlsson, G., and Edwards, K. (2011) Intrinsic heterogeneity in liposome suspensions caused by the dynamic spontaneous formation of hydrophobic active sites in lipid membranes Langmuir, 27(8) 4873-4883.

40. Murzyn, K., Róg, T. and Pasenkiewicz-Gierula M. (2005)

Phosphatidylethanolamine-Phosphatidylglycerol Bilayer as a Model of the Inner Bacterial Membrane. Biophysical Journal, 88(2): 1091-1103.

41. Zinser E., Sperka-Gottlieb C. D., Fasch E. V., Kohlwein S. D., Paltauf F., and Daum G. (1991) Phospholipid synthesis and lipid composition of subcellular membranes in the unicellular eukaryote Saccharomyces cerevisiae Journal of Bacteriology, 173(6): 2026-2034.

42. Doktorova M., Heberle F.A., Eicher B., Standaert R.F., Katsaras J., London E., Pabst G. and Marquardt D. (2018) Preparation of asymmetric phospholipid vesicles for use as cell membrane models. Nature Protocols, 13, 2086-2101.

43. Takaoka R., Kurosaki H., Nakao H., Ikeda K. and Nakano M. (2018) Formation of asymmetric vesicles via phospholipase D-mediated transphosphatidylation.

Biochimica et Biophysica Acta - Biomembranes, 1860(2): 245-249.

44. Redelmeier T. E., Hope M. J., and Cullis P. R. (1990) On the mechanism of transbilayer transport of phosphatidylglycerol in response to transmembrane $\mathrm{pH}$ gradients. Biochemistry, 29(12): 3046:3053.

45. Zakharian, E. (2013) Recording of ion channel activity in planar lipid bilayer experiments Methods in Molecular Biology, 998: 109-118.

46. Oiki, S. (2012) Planar Lipid Bilayer Method for Studying Channel Molecules/n: Patch Clamp Techniques: From Beginning to Advanced Protocols. Springer, Tokyo, 229-275. 
47. Reeves J.P. and Dowben R.M. (1969) Formation and properties of thin-walled phospholipid vesicles Journal of Cellular Physiology, 73(1): 49-60.

48. Stein H., Spindler S., Bonakdar N., Wang C. and Sandoghdar V. (2017) Production of Isolated Giant Unilamellar Vesicles under High Salt Concentrations Frontiers in Physiology, 8: 63.

49. Méléard P., Bagatolli L.A. and Pott T. (2009) Giant Unilamellar Vesicle Electroformation: From Lipid Mixtures to Native Membranes Under Physiological Conditions Methods in Enzymology, 465: 161-176.

50. Conde-Vancells J., Eva Rodriguez-Suarez E., Embad N., Gil D., Matthiesen R., Valle M., Elortza F., Lu, S.C., Mato, J.M. and Falcon-Perez J.M. (2009) Characterization and Comprehensive Proteome Profiling of Exosomes Secreted by Hepatocytes Journal of Proteome Research, 7(12): 5157-5166.

51. Epand, R. M., and Ruysschaert, J-M. (2017) The Biophysics of Cell Membranes: Biological Consequences. Springer Series in Biophysics.

52. Middleton, E. R., and Rhoades, E. (2010) Effects of Curvature and Composition on $\alpha$-Synuclein Binding to Lipid Vesicles Biophys J. 99(7): 2279-2288.

53. Gallier, S., Laubscher, L. Jiménez-Flores, R. (2014) Chapter 4 - The Milk Fat Globule Membrane: Structure, Methodology for its Study, and Functionality. Food Structures, Digestion and Health. 107-142

54. Pick U. (1981) Liposomes with a large trapping capacity prepared by freezing and thawing of sonicated phospholipid mixtures. Archives of Biochemistry and Biophysics, 212(1): 186-194.

55. Costa A.P., Xu X. and Burgess, D.J. (2014) Freeze-Anneal-Thaw Cycling of Unilamellar Liposomes: Effect on Encapsulation Efficiency Pharmaceutical Research, 31:97-103.

56. Patil Y.P. and Jafhav S. (2014) Novel methods for liposome preparation Chemistry and Physics of Lipids, 177: 8-18.

57. Wagner A. and Vorauer-Uhl K. (2011) Liposome Technology for Industrial Purposes. Journal of Drug Delivery, 591325.

58. Hupfeld, S., Holsæter, A.M., Skar, M., Frantzen, C.B., and Brandl, M. (2006) Liposome Size Analysis by Dynamic/Static Light Scattering upon Size Exclusion-/Field Flow-Fractionation Journal of Nanoscience and Nanotechnology, 6(9): 3025-3031.

59. Bhattacharjee, S. (2016) DLS and zeta potential - What they are and what they are not? Journal of Controlled Release, 235:337-351. 
60. Stetefeld, J., McKenna, S.A., and Patel, T.R. (2016) Dynamic light scattering: a practical guide and applications in biomedical sciences Biophys Rev, 8(4): 409-427.

61. Liu, Y. and Liu, J. (2017) Zn ${ }^{2+}$ Induced Irreversible Aggregation, Stacking, and Leakage of Choline Phosphate Liposomes Langmuir, 33(50): 14472-14479.

62. Kahveci, Z., Martínez-Tomé, M.J., Mallavia, R., and Mateo, C.R. (2013) Use of the

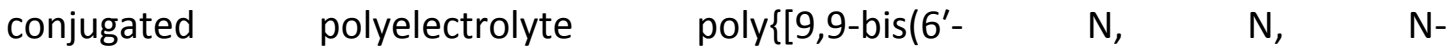
trimethylammonium)hexyl]fluorene-phenylene\} bromide (HTMA-PFP) as a fluorescent membrane marker Biomacromolecules, 14(6): 1990-1998.

63. Soler, M., Estevez, M.C., Villar-Vazquez, R., Casal, J.I., and Lechuga, L.M. (2016) Label-free nanoplasmonic sensing of tumor-associate autoantibodies for early diagnosis of colorectal cancer Analytica Chimica Acta, 930 31-38.

64. Jackman, J.A., Rahim Ferhan, A., and Cho, N.-J. (2017) Nanoplasmonic sensors for biointerfacial science Chem. Soc. Rev., 46(12): 3615-3660.

65. Ferhan, A. R., Ma, G. J., Jackman, J. A., Sut, T. N., Park, J. H., and Cho, N. J. (2017) Probing the interaction of dielectric nanoparticles with supported lipid membrane coatings on nanoplasmonic arrays Sensors (Switzerland), 17(7).

66. Oh, E., Jackman, J.A., Yorulmaz, S., Zhdanov, V.P., Lee, H., and Cho, N.J. (2015) Contribution of temperature to deformation of adsorbed vesicles studied by nanoplasmonic biosensing Langmuir, 31(2): 771-781.

67. Anker, J.N., Hall, W.P., Lyandres, O., Shah, N.C., Zhao, J., and Van Duyne, R.P. (2008) Biosensing with plasmonic nanosensors Nat Mater, 7(6):442-53.

68. Larsson, E.M., Syrenova, S., Langhammer, C., and Giessen, H. (2012) Nanoplasmonic sensing for nanomaterials science Nanophotonics Science Wise Publishing \& De Gruyter@BULLET Berlin @BULLET Boston, 1: 249-266.

69. Jackman, J.A., Avsar, S.Y., Ferhan, A.R., Li, D., Park, J.H., Zhdanov, V.P., and Cho, N.J. (2017) Quantitative profiling of nanoscale liposome deformation by a localized surface plasmon resonance sensor Analytical Chemistry, 89(2): 1102-1109.

70. Langhammer, C., Larsson, E.M., Kasemo, B., and Zorić, I. (2010) Indirect nanoplasmonic sensing: Ultrasensitive experimental platform for nanomaterials science and optical nanocalorimetry Nano Letters, 10(9): 3529-3538.

71. Ferhan, A. R., Jackman, J. A., Malekian, B., Xiong, K., Emilsson, G., Park, S., Dahlin, A. B., and Cho, N. J. (2018) Nanoplasmonic Sensing Architectures for Decoding Membrane Curvature- Dependent Biomacromolecular Interactions Anal. Chem., Just Accepted Manuscript. 
72. Duša, F., Chen, W., Witos' J., and Wiedmer, S. K. (2018) Nanoplasmonic Sensing and Capillary Electrophoresis for Fast Screening of Interactions between Phosphatidylcholine Biomembranes and Surfactants Langmuir, 34(20): 5889-5900.

73. Jonsson, M.P., Jönsson, P., Dahlin, A.B., and Höök, F. (2007) Supported lipid bilayer formation and lipid-membrane-mediated biorecognition reactions studied with a new nanoplasmonic sensor template Nano Letters, 7(11): 3462-3468.

74. Chen, R.F. and Knutson, J.R. (1988) Mechanism of fluorescence concentration quenching of carboxyfluorescein in liposomes: Energy transfer to nonfluorescent dimers Analytical Biochemistry, 172(1) 61-77.

75. Fišer, R. and Konopásek, I. (2009) Different modes of membrane permeabilization by two RTX toxins: HlyA from Escherichia coli and CyaA from Bordetella pertussis Biochimica et Biophysica Acta - Biomembranes, 1788(6): 12491254.

76. Ambroggio, E.E., Separovic, F., Bowie, J.H., Fidelio, G.D., and Bagatolli, L.A. (2005) Direct visualization of membrane leakage induced by the antibiotic peptides: Maculatin, citropin, and aurein Biophysical Journal, 89(3): 1874-1881.

77. Burke, C., Dreher, M. R., Negussie, A. H., Mikhail, A. S, Yarmolenko, P., Patel, A., Skilskyj, B., Wood, B., J and Haemmerich, D. (2018) Drug release kinetics of temperature sensitive liposomes measured at high-temporal resolution with a millifluidic device. International Journal of Hyperthermia, 34;6.

78. Torchilin, V. P., Rammohan, R., Weissig, V., and Levchenko, T. S. (2001) TAT peptide on the surface of liposomes affords their efficient intracellular delivery even at low temperature and in the presence of metabolic inhibitors PNAS 98 (15) 87868791.

79. Peyret, A., Ibarboure, E., Pippa, N., and Lecommandoux, S. (2017) Liposomes in Polymersomes: Multicompartment System with Temperature-Triggered Release Langmuir 22;28 709-7085.

80. Eales, M.G., Ferrarie, E., Goddard, A.D., Lancaster, L., Sanderson, P. and Miller, C. (2018) Mechanistic and phenotypic studies of bicarinalin, BP100 and colistin action on Acinetobacter baumannii Research in Microbiology, pii: S0923-2508(18)30061-5.

81. Ladokhin, A.S., Wimley, W.C., and White, S.H. (1995) Leakage of membrane vesicle contents: determination of mechanism using fluorescence requenching Biophysical Journal, 69(5): 1964-1971.

82. Harris, F. M., Best, K. B., Bell, J. D. (2002) Use of laurdan fluorescence intensity and polarization to distinguish between changes in membrane fluidity and phospholipid order Biochim Biophys Acta, 1565:123-128. 
83. Amaro, M., Reina, F., Hof, M., Eggelin, C., and Sezgin, E. (2017) Laurdan and Di-4ANEPPDHQ probedifferent properties of the membrane J. Phys. D: Appl. Phys, 50: 134004.

84. Sameni, S., Malacrida, L., Tan, Z., and Digman, M. A. (2018) Alteration in Fluidity of Cell Plasma Membrane in Huntington Disease Revealed by Spectral Phasor Analysis Scientific Reports, 8(734).

85. Stott, B. M., Vu, M. P., McLemore, C. O., Lund, M. S., Gibbons, E., Brueseke, T. J., Wilson-Ashworth, H. A., Bell, J. D. (2008) Use of fluorescence to determine the effects of cholesterol on lipid behavior in sphingomyelin liposomes and erythrocyte membranes J Lipid Res, 49(6): 1202-1215.

86. Bouvrais H., Pott T., Bagatolli L.A., Ipsen J.H. and Méléard P. (2010) Impact of membrane-anchored fluorescent probes on the mechanical properties of lipid bilayers Biochimica et Biophysica Acta, 1798(7): 1333-1337.

87. Webb, R.H. (1999) Theoretical basis of confocal microscopy Methods in Enzymology, 307: 3-20.

88. Dal Molin, M., Verolet Q., Colom A., Letrun R., Derivery E., Gonzalez-Gaitan M., Vauthey E., Roux A., Sakai N. and Matile S. (2015) Fluorescent Flippers for Mechanosensitive Membrane Probes Journal of the American Chemical Society, 137(2): 568-571.

89. Licari G., Beckwith J.S., Soleimanpour S., Matile S. and Vauthey E. (2018) Detecting order and lateral pressure at biomimetic interfaces using a mechanosensitive second-harmonic-generation probe Physical Chemistry Chemical Physics, 20: 9328-9336

90. Colom A., Derivery E.,Soleimanpour S., Tomba C., Dal Molin M., Sakai N., González-Gaitán M., Matile S. and Roux A. (2018) A fluorescent membrane tension probe Nature Chemistry, 10: 1118-1125.

91. Robson A., Dastoor P.C., Flynn J., Palmer W., Martin A., Smith D.W., Woldu A. and Hua S. (2018) Advantages and Limitations of Current Imaging Techniques for Characterizing Liposome Morphology Frontiers in Pharmacology, 9: 80.

92. Kuntsche J., Horst J.C. and Heike B. (2011) Cryogenic transmission electron microscopy (cryo-TEM) for studying the morphology of colloidal drug delivery systems International Journal of Pharmaceuticals, 417(1-2): 120-137.

93. Bibi S., Kaur R., Henriksen-Lacey M., McNeil S.E., Wilkhu J., Lattmann E., Christensen D., Mohammed A.R. and Yvonne Perrie Y. (2011) Microscopy imaging of liposomes: From coverslips to environmental SEM International Journal of Pharmaceuticals, 417(1-2): 137-150. 
94. De Carlo S., Fiaux H. and Marca-Martinet C.A. (2004) Electron Cryo-Microscopy Reveals Mechanism of Action of Propranolol on Artificial Membranes Journal of Liposome Research 14(1-2): 61-76.

95. Chiu M.H. and Prenner E.J. (2011) Differential scanning calorimetry: An invaluable tool for a detailed thermodynamic characterization of macromolecules and their interactions Journal of Pharmacy and BioAllied Sciences, (1): 39-59.

96. Demetzos C. (2008) Differential Scanning Calorimetry (DSC): a tool to study the thermal behavior of lipid bilayers and liposomal stability. Journal of Liposome Research, 18(3): 159-173.

97. Sturtevant J.M (1987) Biochemical Applications of Differential Scanning Calorimetry Annual Review of Physical Chemistry, 38: 463-488.

98. Higashino Y., Matsui A. and Ohki K. (2001) Membrane fusion between liposomes composed of acidic phospholipids and neutral phospholipids induced by melittin: a differential scanning calorimetric study. Journal of Biochemistry, 130(3): 393-397.

99. Komorowski K., Salditt A., Xu Y., Yavuz H., Brennich M., Jahn, R. and Salditt T. (2018) Vesicle Adhesion and Fusion Studied by Small-Angle X-Ray Scattering Biophysical Journal, 114(8): 1908-1920.

100. Garcia-Diez R., Gollwitzer C., Krumrey M. and Varga Z. (2016) Size Determination of a Liposomal Drug by Small-Angle X-ray Scattering Using Continuous Contrast Variation. Langmuir, 32(3): 772-778.

101. Aburai K., Ogura T., Hyodo R., Sakai H., Abe M., and Glatter O. (2013) Location of cholesterol in liposomes by using small-angle X-ray scattering (SAXS) data and the generalized indirect Fourier transformation (GIFT) method. Journal of Oleo Science, 62(11): 913-918.

\section{Figure Legends}

Figure 1: Carpet mechanism of membrane disruption by antimicrobial peptides. A) peptides bind to the membrane and $B$ ) accumulate and cover the membrane before C) disrupting it into smaller sections.

Figure 2: Liposome preparation. Liposomes are prepared by evaporating organic solvent from the lipids, rehydrating it with the appropriate buffer to the desired concentration. The lipid is then extruded repeatedly through filters of the desired pore size (ranging from $0.1 \mu \mathrm{m}$ to $12 \mu \mathrm{m}$ ) to form small unilamellar liposomes.

Figure 3: Dynamic Light Scattering. A monochromatic beam of light is fired through a sample, and particles within the sample scatter the light. Changes in intensity of the scattered light are measured to determine Brownian motion and used to obtain 
the diameter of the particles.

Figure 4: Nanoplasmonic sensing. Samples such as intact liposomes or bilayers are adsorbed onto $\mathrm{SiO}_{2}$-coated gold nanodisks supported on a glass sensor. Structural information about the adsorbed molecules can be obtained by monitoring changes to $\Delta \lambda_{\max }$.

Figure 5: Carboxyfluorescein dye leakage assay. Liposomes are rehydrated with 100 $\mathrm{mM}$ carboxyfluorescein and extruded. CF self-quenches at high concentrations within the liposome; upon treatment with a disrupting compound, the CF is released and diluted, resulting in an increase in fluorescence 
A

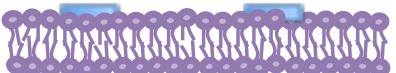

B

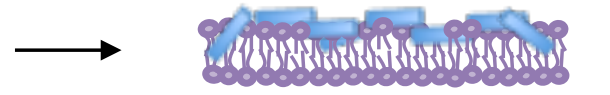

C

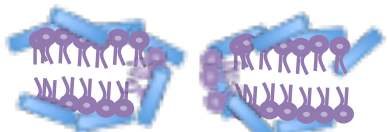


Evaporate organic solvent to produce lipid film
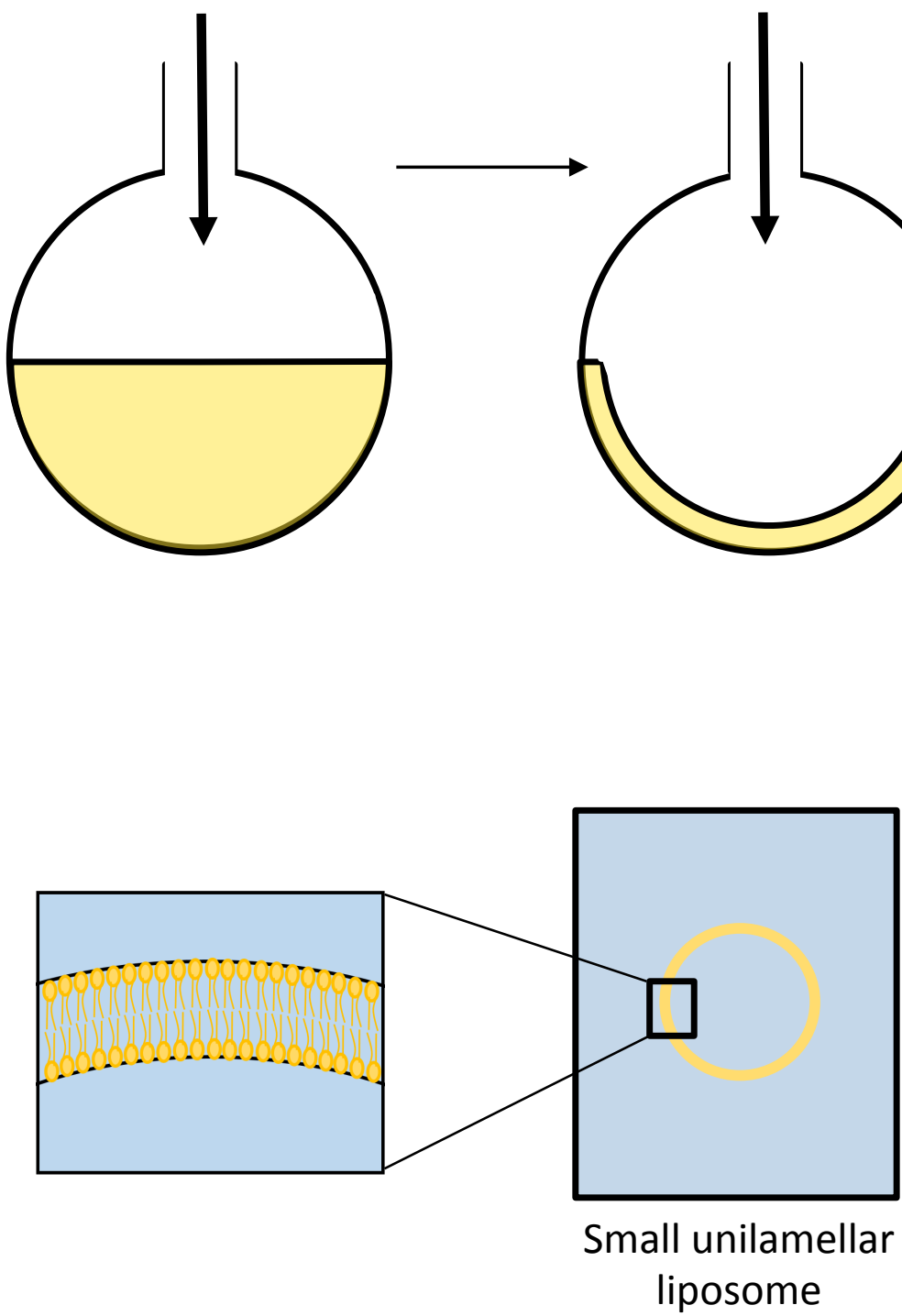

Rehydrate film with buffer/

dye; vortex

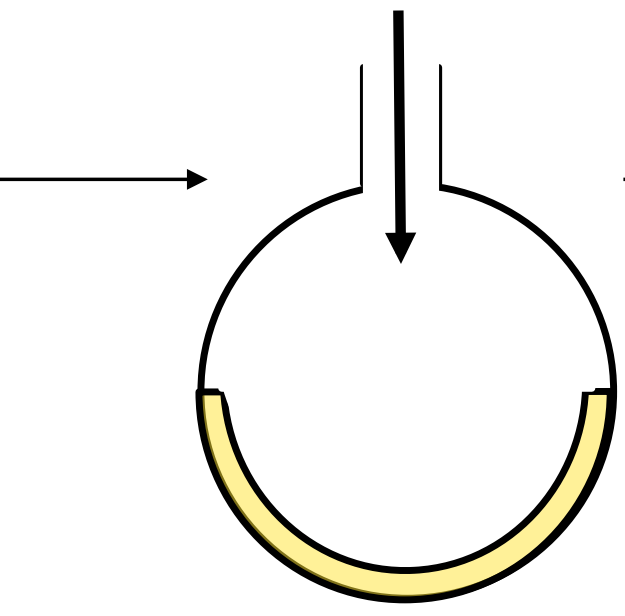

Extrusion

Sonication

Sonication 


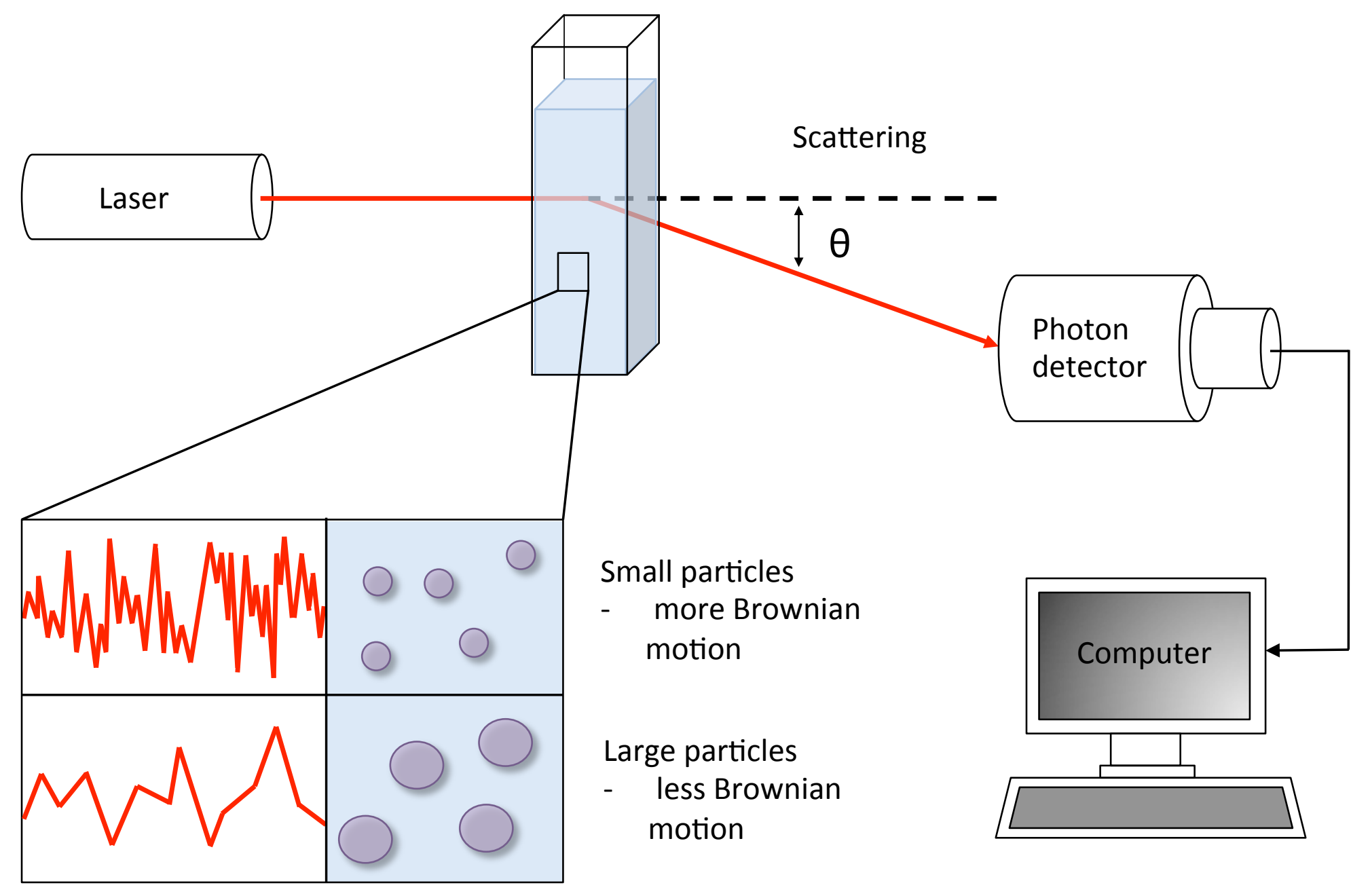


Incident light

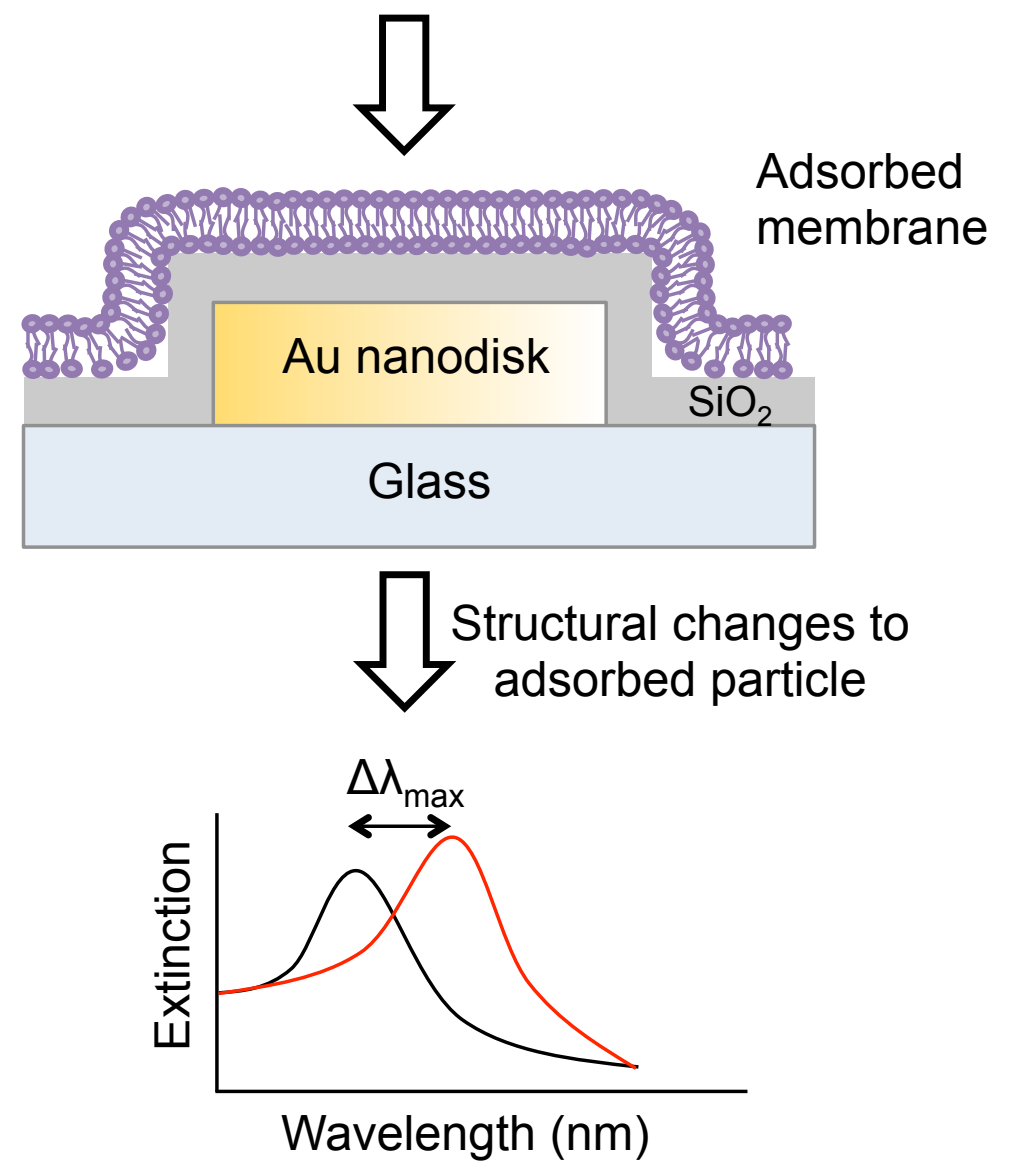




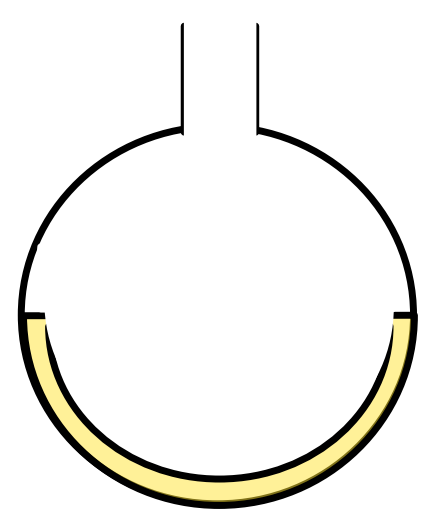

Rehydrate lipid film in CF and extrude

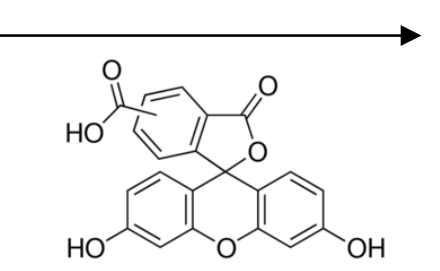

5(6)-Carboxyfluorescein (CF)



$$
\begin{gathered}
\text { CF at high } \\
\text { concentration inside } \\
\text { liposome }
\end{gathered}
$$
compound

\author{
Measure change in \\ fluorescence: \\ Excitation at $492 \mathrm{~nm}$ \\ Emission at $517 \mathrm{~nm}$
}

